\title{
Can we distinguish between inflammatory and neuropathic pain?
}

\author{
Gary J Bennett PhD
}

\begin{abstract}
GJ Bennett. Can we distinguish between inflammatory and neuropathic pain? Pain Res Manage 2006;11(Suppl A):11A-15A.

Inflammatory and neuropathic pain were once considered to be distinct entities. However, research over the past decade or so has brought to light many shared mechanisms, and the distinction between the two is no longer clear. Consideration of mechanisms, symptoms and the effects of analgesic drugs does not reveal any definitive or universally applicable differentiating factors. Given the present level of understanding, it may not be possible to distinguish between inflammatory and neuropathic pain in a large number of patients, and a satisfying definition of neuropathic pain may not be possible.
\end{abstract}

Key Words: Allodynia; Chronic pain; Hyperalgesia; Inflammation; Neuropathy

\section{Peut-on distinguer la douleur inflammatoire de la douleur neuropathique?}

La douleur inflammatoire et la douleur neuropathique ont déjà été considérées comme des entités distinctes. Cependant, les recherches depuis une dizaine d'années ont mis en lumière de nombreux mécanismes partagés, et la distinction entre les deux types de douleur n'est plus claire. Lévaluation des mécanismes, des symptômes et des effets des analgésiques ne révèle aucun facteur différentiel universellement applicable. Étant donné le niveau de compréhension actuel, il n'est peut-être possible ni de distinguer la douleur inflammatoire de la douleur neuropathique chez un grand nombre de patients, ni de parvenir à une définition satisfaisante de la douleur neuropathique.
The distinction between inflammatory and neuropathic pain used to seem quite clear. But, as our understanding of peripheral and central pain mechanisms has increased during the past decade or so, the distinction has become markedly less clear. As a result, we find current debates about the definition of neuropathic pain, and the debate is fueled by attempts to determine whether certain clinical entities (eg, complex regional pain syndrome type I [CRPS-I], fibromyalgia and interstitial cystitis) should be classified as neuropathic (1-5). I do not have a solution to this problem, but I will try here to delineate what I think are the key problems that must be addressed in seeking a solution.

To begin, exactly what kinds of pain syndromes are we speaking about? There is little reason to suspect that acute nociceptive pain (stepping on a tack) and neuropathic pain share important mechanistic factors. It is the mechanisms operating in inflammatory pain that sometimes seem similar to those of neuropathic pain. Moreover, there is little evidence to suggest that central neuropathic pain (ie, the neuropathic pain that follows injury to the somatosensory regions of the central nervous system) shares any significant mechanism with inflammatory pain. Thus, the controversy refers specifically to neuropathic pain as it presents in the various kinds of painful peripheral neuropathies and inflammatory pain (chronic, rather than acute, inflammatory pain in particular). For brevity's sake, I will refer to these as 'inflammatory' pain and 'neuropathic' pain.

\section{WHAT CONSTITUTES A NERVE LESION?}

If neuropathic pain is defined as being caused by a nerve lesion, then differentiating it from inflammatory pain will depend on our ability to document a nerve lesion. Our ability to do this is not very impressive. Trauma or disease that injured $50 \%$ of the axons in the sciatic nerve would be easy to document and would obviously qualify as a nerve injury. But injury to $5 \%$ of the axons in the sciatic nerve would not be easy to document. The gold standard electrodiagnostic procedure, the sensory nerve conduction velocity (SNCV) test, is insensitive to partial injuries and to injuries to small $\mathrm{A}$ - and C-fibres. A sciatic nerve in which every single $\mathrm{C}$-fibre had died could have a perfectly normal SNCV. Many nerves are small; for example, the knee joint is innervated by several small nerves and it is probable that one of these is sometimes damaged in arthroscopic procedures. Such damage would be difficult to document, because the SNCV tests cannot be performed on small, deep, short nerves. In some circumstances, quantitative sensory testing (QST) may be used to document small or partial nerve injuries, or injuries to just small fibres. But such documentation is accepted only if one allows 'subjective' evidence. Moreover, the sensitivity of QST for small nerve injuries has not been documented, and it is not always practical (how would one use QST to detect damage to the knee joint's innervation?).

As they approach their target tissue, somatosensory nerves divide into smaller and smaller branches; the smallest, most 
distal visible branches are often called fascicles rather than nerves, but this obscures the fact that but all but the very last of the successive branches are properly called nerves (ie, a bundle of axons surrounded by a connective tissue sheath, the epineurium). As the smallest nerves divide further, the epineurium disappears and the individual axons travel in small bundles; these are properly defined as fascicles. It is approximately at this stage that the structures become too small to see with the naked eye. Individual sensory axons leave their fascicle and penetrate into the tissue that they innervate. In the skin, for example, C-nociceptors leave their fascicles in the dermis and cross into the epidermis, where they branch and issue their receptor terminals throughout the epidermis up to the stratum corneum (the superficial layer of dead keratinocytes). These intraepidermal branches and their receptor terminals are the sensory neuron's terminal arbor.

In densely innervated skin, such as the human finger tip, it is probably impossible to stick a needle in the skin without damaging terminal arbors. Is this a nerve injury? Is damage to a fascicle a nerve injury? Is damage to the smallest nerve branches with an epineurium a nerve injury? Even extensive damage to these small structures are difficult to document. Microscopic examination of skin biopsies from CRPS-I patients has shown a partial but statistically significant loss of intraepidermal terminal arbors $(6,7)$. Similar examinations of biopsies of the tongue's epithelium have shown a clear loss of sensory terminal arbors in patients with burning mouth syndrome (glossodynia; 8). Siau et al (9) have recently found a similar loss of intraepidermal terminal arbors in rats with painful peripheral neuropathies due to the antineoplastic drugs, paclitaxel and vincristine, and neither light- nor electron-microscopic examination of the peripheral nerves in these animals found any evidence of degeneration (10). In the least, one is justified in suspecting that this damage to sensory terminal arbors, even in the absence of damage to the nerve from which their axons come, is the lesion producing pain in CRPS-I, burning mouth syndrome and chemotherapy-evoked peripheral neuropathy, and that these are thus properly classified as neuropathic pain conditions. If this is correct, we will have to use our words carefully in a definition of neuropathic pain: 'pain caused by a lesion to somatosensory neurons, not a nerve lesion'.

\section{WHAT CONSTITUTES INFLAMMATION?}

If inflammatory pain is defined as being caused by inflammation, then differentiating it from neuropathic pain will depend critically on our ability to document inflammation. This is not always easy to do. We are very familiar with the signs and mechanisms of acute inflammation, but we know far less about chronic inflammation. Until fairly recently, there was debate as to whether an inflammatory pain condition existed in the osteoarthritic joint (it does; for review see 11). Chronic low back pain does not usually present with obvious signs of an inflammatory condition, but it now seems likely that at least some patients have a chronic periepidural inflammatory condition mediated by tumour necrosis factor-alpha and other proinflammatory cytokines (12). One might be tempted to say that any pain that was relieved by a nonsteroidal antiinflammatory drug was inflammatory pain by definition. But these drugs work primarily by inhibiting the arachidonic acid cascade, which is only part of the inflammatory process. Nonsteroidal anti-inflammatory drugs have little effect on proinflammatory cytokines. I think we must consider the possibility that our conception of what constitutes inflammation is unclear, and places too heavy an emphasis on acute, rather than chronic, processes and too heavy an emphasis on inflammatory processes in the skin, rather than in muscle, tendon, bone and visceral organs.

\section{PAIN FROM MIXED MECHANISMS}

Inflammation can cause neural damage, and neural damage can cause inflammation. I think it is likely that inflammatory and neuropathic pain mechanisms frequently coexist. This makes the distinction between the two difficult in both the clinical and experimental contexts.

Inflammatory processes damage healthy tissue that surrounds an injury or infection, and this damage includes the tissue's sensory innervation. As noted above, inflammation-evoked damage to even the sensory terminal arbors within the tissue may constitute a lesion capable of producing neuropathic pain. Perhaps the clearest example of this is with bone cancer, where the intramedullary sensory innervation is destroyed by the expanding tumour mass and the inflammatory response to the presence of the tumour (13).

Neural damage from any cause will produce an inflammatory response. Even in a perfectly sterile injury, cellular debris from degenerating nerves, fascicles and terminal arbors is a sufficient stimulus to activate the immune system. This inflammatory response is a necessary component of the healing process; cellular debris must be removed (or at least cordoned off) before tissue regeneration can proceed. Ordinarily, this is an acute process. But one can imagine situations in which it would be chronic, or chronically episodic. For example, nerves have limited elasticity and are generally not attached to surrounding structures; thus, bending the elbow produces a relatively small stretch to the ulnar nerve. A nerve that becomes tethered to adjacent tissue loses much of its elasticity and may suffer excessive stretch every time the surrounding structures moved, resulting in repetitive microdamage that generates cellular debris. This is a likely reason for the pain of patients with 'tennis elbow' or carpal tunnel entrapment. Entrapment of the ulnar nerve is easy to detect, but could we document entrapment of an articular nerve in postarthroscopic scar tissue? As another example, consider the distal, symmetrical, dying-back neuropathy that is so common in the diabetic patient. The cause of the axonal degeneration is unknown, but it is clearly a chronic and progressive process, in which new neural debris is constantly produced. It is thus conceivable that the diabetic patient's nerve is constantly inflamed (and that inflammation itself may contribute to axonal injury).

It is particularly noteworthy that the incidence of pain following inflammation and neural injury is clearly different. Any but the most trivial inflammatory condition is accompanied by at least some pain in nearly everyone (the only important exception being when the inflammation is confined to an organ that does not have a sensory innervation, eg, the brain). But neural damage causes pain in only a minority of patients. For example, only approximately 20\% of diabetics with peripheral neuropathy have neuropathic pain. The highest incidence is approximately $50 \%$ for postherpetic neuralgia patients who have shingles when they are in their 80 s or older. Why apparently identical neural lesions cause neuropathic pain in some people but not in others is one of the outstanding questions. I 
suspect that differentiating neuropathic and inflammatory pain would be easier if we had the answer.

\section{THE SPECIAL CASE OF PAIN DUE TO NEURITIS}

'Neuritis' refers to an inflamed nerve, but the importance of the exact location of the inflammation is rarely considered. Inflammation of the nerve's epineurial sheath will activate and sensitize nociceptors that are part of the sheath's sensory innervation (ie, the nervi nervorum). The result is likely to be a nerve that is sore and tender to palpation, and the pain is likely to be fairly well localized. If one asks a patient with sciatica to point to his pain, one will often see the patient press his finger into the sciatic notch, drag it across the buttock, press it between the medial and lateral thigh muscles on the back of his leg, and run the finger down between the muscles to the popliteal fossae. I suggest that the patient is tracing the course of his sciatic nerve. His nerve is sore; to be precise, his epineurium is sore. Such pain would be classified as inflammatory pain.

However, an inflammatory process need not be confined to the nerve's connective tissue sheath - it may also be present in the endoneurial compartment. The sensory axons travelling within a nerve do not express the transduction molecules that are responsible for activation by noxious stimuli. These molecules are expressed only in the membrane of the sensory axon's terminals (alternatively, they may be expressed in the axonal membrane everywhere, but functional only when inserted into the terminal's membrane). However, there is evidence that at least some inflammatory mediators can evoke an ectopic activation of the axon itself. For example, normal C-nociceptor axons will discharge when tumour necrosis factor-alpha is applied endoneurially (14). Pain produced in this way will not be localized to the nerve; it will be felt in the tissue innervated by the activated axon. It seems reasonable to classify this as neuropathic pain.

Thus, I propose a distinction between epineurial neuritis and endoneurial neuritis, with the former producing inflammatory pain and the latter producing neuropathic pain. Clinically, one might expect to find either in relative isolation in at least some cases, but one can easily imagine that they commonly coexist. Moreover, there is reason to believe that an epineurial inflammation can evoke an endoneurial inflammation via a mechanism distinct from simple spread across the nerve sheath (15).

\section{SYMPTOMS}

Is it possible to differentiate inflammatory and neuropathic pain on the bases of their symptoms? I think the answer is "sometimes, but not very often". First, we must acknowledge that our knowledge of exactly what kinds of pain abnormality are present in inflammatory and neuropathic pain patients is woefully inadequate. For example, what percentage of patients with painful diabetic peripheral neuropathy have cutaneous mechanoallodynia? Do osteoarthritis patients have heathyperalgesia in the skin overlying their painful joints? Is an attack of gout accompanied by cutaneous mechanoallodynia? I think the answer to both questions is yes, but little evidence is available (16).

Second, it is important to note that the location of the symptoms usually differs. Cutaneous pain abnormalities are frequent in neuropathic pain patients, but relatively rare in patients with inflammatory pain. The exceptions to this are the relatively rare cases with cutaneous inflammatory disease and the more common cases of cutaneous referred pain secondary to inflammation of some visceral organs. Chronic inflammatory pain is most often localized to musculoskeletal and visceral tissue (eg, osteoarthritis and cystitis). Neuropathic pain patients sometimes report deep tissue pain, usually in muscle and bone, but this deep tissue pain has received far less study than the cutaneous symptoms and its incidence is unknown. Importantly, experimental investigations in both animals and patients have mostly looked only at abnormalities of cutaneous pain. Neuropathic pain referred to a visceral organ is either exceedingly rare or not convincingly documented. This is noteworthy, given that surgical injuries to sensory nerves innervating visceral organs (eg, excision of a gall bladder or uterus) are common.

Third, the complaints of patients with inflammatory pain are relatively uniform (arthritis patients have aching pain in their joints, low-back pain patients have aching pain in their backs, regardless of whether they have sciatica) and their pain seems well localized to the area of inflammation. In contrast, neuropathic pain patients present with a bewildering mix of symptoms such as burning pain, aching pain, stabbing pain, mechanoallodynia, cold allodynia and heat hyperalgesia, alone or in multiple combinations. Moreover, neuropathic pain is frequently not well localized and may spread to the territories of multiple nerves and dermatomes (17). However, localization of pain is unlikely to be a key differentiating factor for inflammatory and neuropathic pain.

There are some differences in the symptoms. Cold allodynia is a frequent (although far from invariant) symptom in at least some kinds of neuropathic pain. The cold-pain threshold depends strongly on the size of the area stimulated, but is generally in the range of $0^{\circ} \mathrm{C}$ to $10^{\circ} \mathrm{C}$ in normal persons. Neuropathic pain patients with cold allodynia report pain with stimuli of $15^{\circ} \mathrm{C}$ to $20^{\circ} \mathrm{C}$, and it is important to note that the pain that is evoked has an abnormal quality - 'burning', rather than the 'aching' pain that cold normally evokes. I am not aware of any demonstration that cold allodynia exists in patients with inflammatory pain. Indeed, cold relieves inflammatory pain. It is thus possible that the presence of cold allodynia is an unequivocal marker for neuropathic pain, although its absence clearly does not preclude the presence of neuropathic pain.

Some neuropathic pain patients report shooting, electric shock-like pains. I have argued elsewhere (18) that such paroxysmal pains are literally like those produced by an electric shock, because they are both due to the simultaneous activation of large numbers of sensory axons. I am not aware of any purely inflammatory condition where the patient reports electric shock-like pain. It is thus possible that the presence of electric shock-like pain is an unequivocal marker for neuropathic pain, although here again its absence clearly does not preclude the presence of neuropathic pain.

Neuropathic pain is frequently (although not invariably) associated with a partial loss of sensory acuity in the area of the pain. This is probably a correlate of the inciting event - damage to sensory neurons. However, a partial loss of sensory acuity has also been reported for inflammatory pain conditions, where it has been hypothesized as being due to changes in central processing (16). Thus, it seems that the presence or absence of a decrease in sensory acuity will not be a clear differentiating factor. 
Dysesthetic sensations are commonly reported by neuropathic pain patients. By definition, these are not pain. Patients frequently volunteer that dysesthetic sensations are difficult to describe. The word 'numb' is often used, not in the doctor's sense of 'insensate', but in the common usage of the word to describe the positive sensations arising from an ischemic limb. The presence of dysesthetic sensations may be an excellent differentiating factor for neuropathic versus inflammatory pain (19), but its absence will not exclude the presence of neuropathic pain.

\section{TREATMENT RESPONSES}

Is it possible to differentiate inflammatory and neuropathic pain on the bases of their responses to drugs? I think the answer is probably not.

Until recently, one might have asserted that pain that was resistant to opioids was neuropathic pain by definition. This is no longer tenable - neuropathic pain can be relieved by opioids, although it is very likely that relief is obtained only with doses larger than those commonly used for the relief of inflammatory pain and that their efficacy against stimulus-evoked neuropathic pain may be limited $(20,21)$.

Tricyclic antidepressants (TCAs) are a mainstay in the treatment of neuropathic pain and it is clear that their efficacy is separable from their effects on mood (22). Accumulating evidence suggests that the efficacy of TCAs against neuropathic pain may result at least in part from their ability to block voltagegated sodium channels (23). But animal studies suggest that TCAs may have at least some efficacy against inflammatory pain and open-label trials suggest that sodium channel blockers may also be effective in patients with osteoarthritis and low-back pain $(24,25)$. It is noteworthy that at least some of the changes in sodium channel expression in primary afferent neurons that were first found in neuropathic pain models are now known to also occur in chronic inflammatory pain conditions (26). Thus, analgesic effects from TCAs and sodium channel blockers will probably not be a clear differentiating factor for neuropathic and inflammatory pain.

Several of the new generation of antiepileptic drugs have efficacy in at least some neuropathic pain patients. The best characterized drugs in this group are gabapentin and pregabalin (gabapentinoids). Gabapentinoids have little or no analgesic effects in animal models of inflammatory pain and I am not aware of any unequivocal evidence that they have analgesic activity in patients with inflammatory pain (it may simply be that no one has looked). However, very recent reports suggest that gabapentinoids may have efficacy in preventing and reducing at least some types of postoperative pain $(27,28)$.

\section{REFERENCES}

1. Backonja MM. Defining neuropathic pain. Anesth Analg 2003;97:785-90. (Erratum in 2004;98:67)

2. Bennett GJ. Neuropathic pain: A crisis of definition? Anesth Analg 2003;97:619-20.

3. Attal N, Bouhassira D. Can pain be more or less neuropathic? Pain 2004;110:510-1.

4. Rasmussen PV, Sindrup SH, Jensen TS, Bach FW. Symptoms and signs in patients with suspected neuropathic pain. Pain 2004;110:461-9.

5. Committee on classification and taxonomy. Special Interest Group on Neuropathic Pain of the International Association for the Study of Pain. Neuropathic pain. Newsletter, 2006;7<www.neupsig.org/> (Version current at April 26, 2006).

6. Albrecht PJ, Hines S, Eisenberg E, et al. Pathologic alterations of cutaneous innervation and vasculature in affected limbs from patients with complex regional pain syndrome. Pain 2006;120:244-66.
It seems clear that gabapentinoids do not work in all neuropathic pain patients; it does not work in all rats with painful peripheral neuropathy either. There is evidence suggesting that the efficacy of gabapentinoids depends on whether there is a significant injury-evoked increase in calcium channels that express its binding site, the $\alpha_{2} \delta$ type-1 subunit $(29,30)$. It is not known whether chronic inflammation is accompanied by a similar increase in channel subunit expression. In general, neuronal voltage-gated calcium channels are essential for the exocytosis of synaptic vesicles and, hence, are key regulators of neurotransmission. Thus, any drug that blocks these channels would be expected to have efficacy against both inflammatory and neuropathic pain (31).

\section{CONCLUSIONS}

In both conceptual and, especially, practical terms, it is very difficult to make a clear distinction between neuropathic and inflammatory pain. The more experiments we do, the more similar inflammatory and neuropathic pain seem to be. Clear differentiating factors are not present with respect to mechanisms, symptoms or treatment responses. At least some central mechanisms are evoked when $\mathrm{C}$-nociceptors discharge, and it is difficult to see why the central response would care whether the discharge was due to products of the arachidonic acid cascade or to sodium channels accumulating at the tip of a severed axon. What were once clear cut differences now seem to rest more on ignorance than fact. The pain-producing mechanisms associated with chronic inflammation are very poorly understood. Drugs that are 'specific' for neuropathic pain have rarely been tested in patients with inflammatory pain. Inflammation and neural injury may be inextricably intertwined, especially if injury to sensory terminal arbors is mechanistically important.

Perhaps we are faltering because we are presenting the problem as a comparison between two very generic terms. It is becoming very clear that there are a large number of mechanisms underlying the various kinds of pain abnormalities seen in peripheral neuropathy $(18,32)$. An equally large number may underlie inflammatory pain. Perhaps we would make more progress by trying to differentiate, for example, inflammatory cutaneous mechanoallodynia from neuropathic cutaneous mechanoallodynia.

For now, I think that we will have to live with definitions that are far from ideal, and live with the fact that many patients will have pain conditions that are not easily classified. We simply must know more before we can solve the problem.

7. Oaklander AL, Rissmiller JG, Gelman LB, Zheng L, Chang Y, Gott R. Evidence of focal small-fiber axonal degeneration in complex regional pain syndrome-I (reflex sympathetic dystrophy). Pain 2006;120:235-43.

8. Lauria G, Majorana A, Borgna M, et al. Trigeminal small-fiber sensory neuropathy causes burning mouth syndrome. Pain 2005; 115:332-7.

9. Siau C, Xiao WH, Bennett GJ. Effects on epidermal innervation density and cutaneous Langerhans cell activation of peripheral nerve injury due to trauma, paclitaxel, vincristine, and dideoxycytidine. Exp Neurol 2006. (In press)

10. Flatters SJ, Bennett, GJ. Studies of peripheral sensory nerves in paclitaxel-induced painful peripheral neuropathy: Evidence for mitochondrial dysfunction. Pain 2006; Epub ahead of print.

11. Felson DT. The sources of pain in knee osteoarthritis. Curr Opin Rheumatol 2005;17:624-8. 
12. Brisby H, Olmarker K, Larsson K, Nutu M, Rydevik B. Proinflammatory cytokines in cerebrospinal fluid and serum in patients with disc herniation and sciatica. Eur Spine J 2002;11:62-6.

13. Peters CM, Ghilardi JR, Keyser CP, et al. Tumor-induced injury of primary afferent sensory nerve fibers in bone cancer pain. Exp Neurol 2005;193:85-100.

14. Sorkin LS, Xiao WH, Wagner R, Myers RR. Tumour necrosis factor-alpha induces ectopic activity in nociceptive primary afferent fibres. Neuroscience 1997;81:255-62.

15. Eliav E, Herzburg U, Ruda MA, Bennett GJ. Neuropathic pain from an experimental neuritis of the rat sciatic nerve. Pain 1999;83:169-82.

16. Hendiani JA, Westlund KN, Lawand N, Goel N, Lisse J, McNearney T. Mechanical sensation and pain thresholds in patients with chronic arthropathies. J Pain 2003;4:203-11.

17. Tal M, Bennett GJ Extra-territorial pain in rats with a peripheral mononeuropathy: Mechano-hyperalgesia and mechano-allodynia in the territory of an uninjured nerve. Pain 1994;57:375-82.

18. Bennett GJ. Neuropathic pain. In: Wall PD, Melzack R, eds. Textbook of Pain, 3rd edn. Edinburgh: Churchill Livingstone, 1994:201-24.

19. Bouhassira D, Attal N, Alchaar H, et al. Comparison of pain syndromes associated with nervous or somatic lesions and development of a new neuropathic pain diagnostic questionnaire (DN4). Pain 2005;114:29-36.

20. Raja SN, Haythornthwaite JA, Pappagallo M, et al. Opioids versus antidepressants in postherpetic neuralgia: A randomized, placebocontrolled trial. Neurology 2002;59:1015-21.

21. Eisenberg E, McNicol ED, Carr DB. Efficacy of mu-opioid agonists in the treatment of evoked neuropathic pain: Systematic review of randomized controlled trials. Eur J Pain 2005.:Epub ahead of print.

22. Kishore-Kumar R, Max MB, Schafer SC, et al. Desipramine relieves postherpetic neuralgia. Clin Pharmacol Ther 1990;47:305-12.

23. Amir R, Argoff CE, Bennett GJ, et al. The role of sodium channels
PNC, = DO NOT COPY

in chronic inflammatory and neuropathic pain. J Pain 2006;7(Suppl 5):S1-S29.

24. Galer BS, Gammaitoni AR, Oleka N, Jensen MP, Argoff CE. Use of the lidocaine patch $5 \%$ in reducing intensity of various pain qualities reported by patients with low-back pain. Curr Med Res Opin 2004;20 (Suppl 2):S5-12.

25. Gammaitoni AR, Galer BS, Onawola R, Jensen MP, Argoff CE. Lidocaine patch $5 \%$ and its positive impact on pain qualities in osteoarthritis: Results of a pilot 2-week, open-label study using the Neuropathic Pain Scale. Curr Med Res Opin 2004;20(Suppl 2):S13-9.

26. Black JA, Liu S, Tanaka M, Cummins TR, Waxman SG. Changes in the expression of tetrodotoxin-sensitive sodium channels within dorsal root ganglia neurons in inflammatory pain. Pain 2004;108:237-47.

27. Al-Mujadi H, A-Refai AR, Katzarov MG, Dehrab NA, Batra YK, Al-Qattan AR. Preemptive gabapentin reduces postoperative pain and opioid demand following thyroid surgery. Can J Anaesth 2006;53:268-73.

28. Sihoe AD, Lee TW, Wan IY, Thung KH, Yim AP. The use of gabapentin for post-operative and post-traumatic pain in thoracic surgery patients. Eur J Cardiothorac Surg 2006: Epub ahead of print.

29. Luo ZD, Calcutt NA, Higuera ES, et al. Injury type-specific calcium channel alpha 2 delta-1 subunit up-regulation in rat neuropathic pain models correlates with antiallodynic effects of gabapentin. J Pharmacol Exp Ther 2002;303:1199-205.

30. Li CY, Song YH, Higuera ES, Luo ZD. Spinal dorsal horn calcium channel alpha2delta-1 subunit upregulation contributes to peripheral nerve injury-induced tactile allodynia. J Neurosci 2004;24:8494-9.

31. Snutch TP. Targeting chronic and neuropathic pain: The $\mathrm{N}$-type calcium channel comes of age. NeuroRx 2005;2:662-70.

32. Woolf CJ, Bennett GJ, Doherty M, et al. Towards a mechanismbased classification of pain? Pain 1998;77:227-9. 


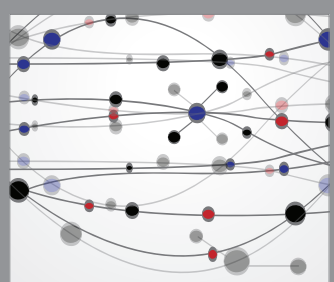

The Scientific World Journal
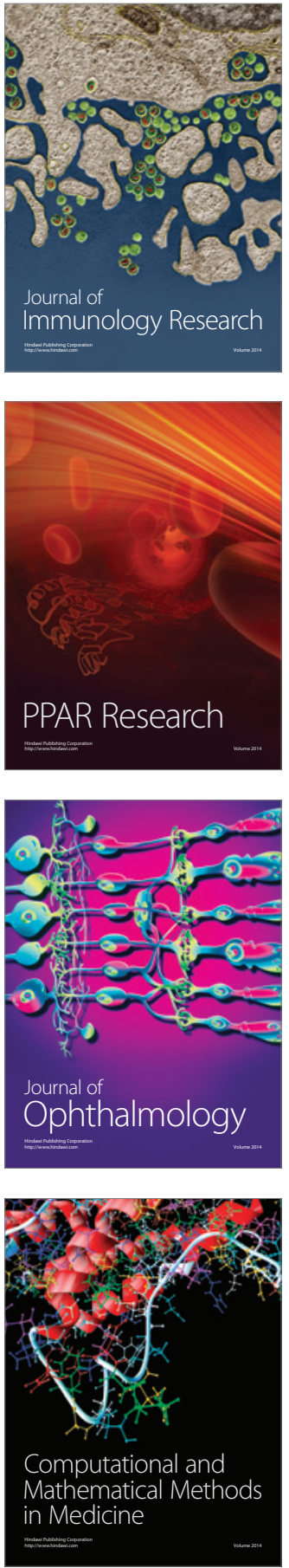

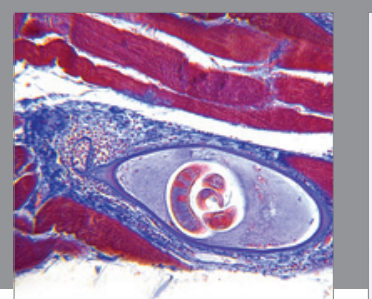

Gastroenterology Research and Practice

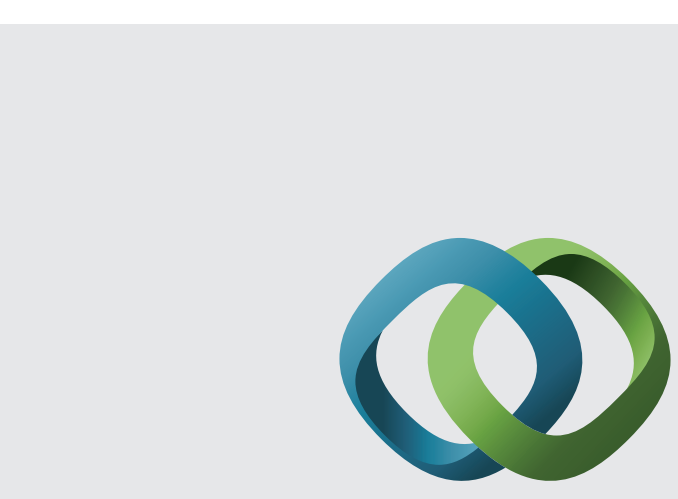

\section{Hindawi}

Submit your manuscripts at

http://www.hindawi.com
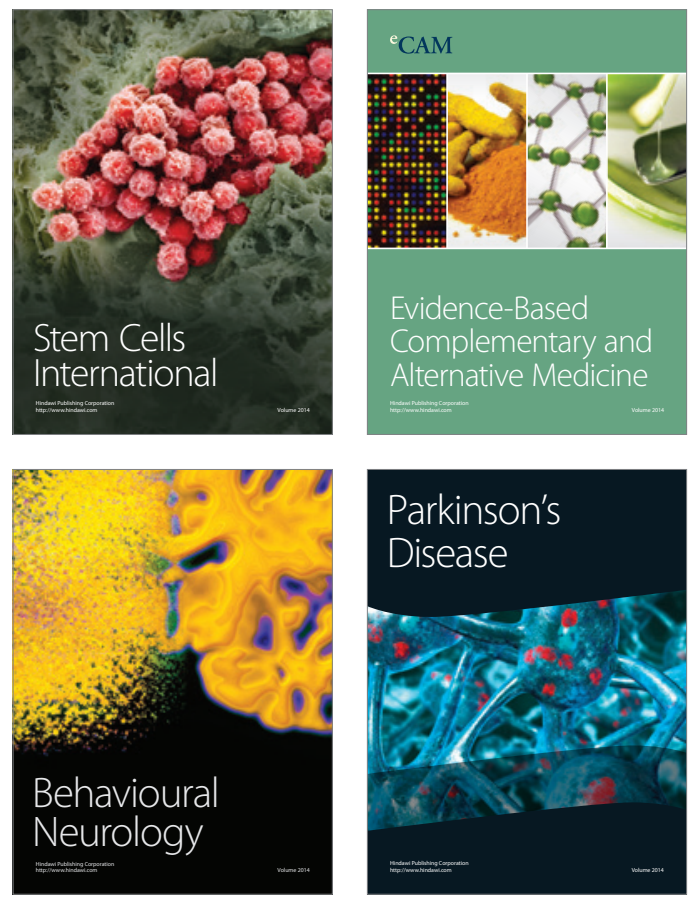
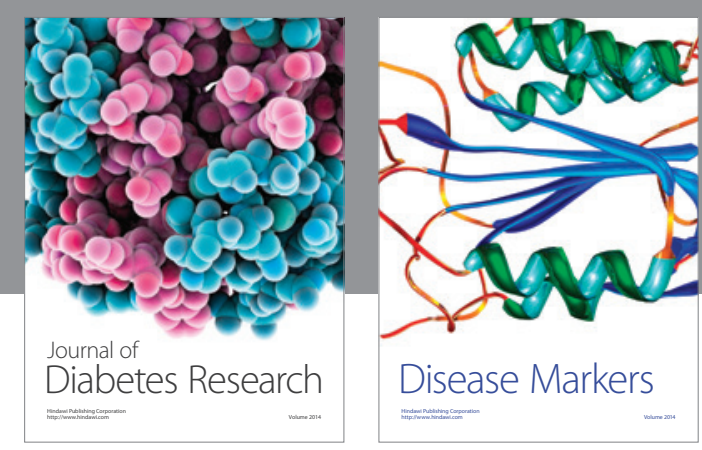

Disease Markers
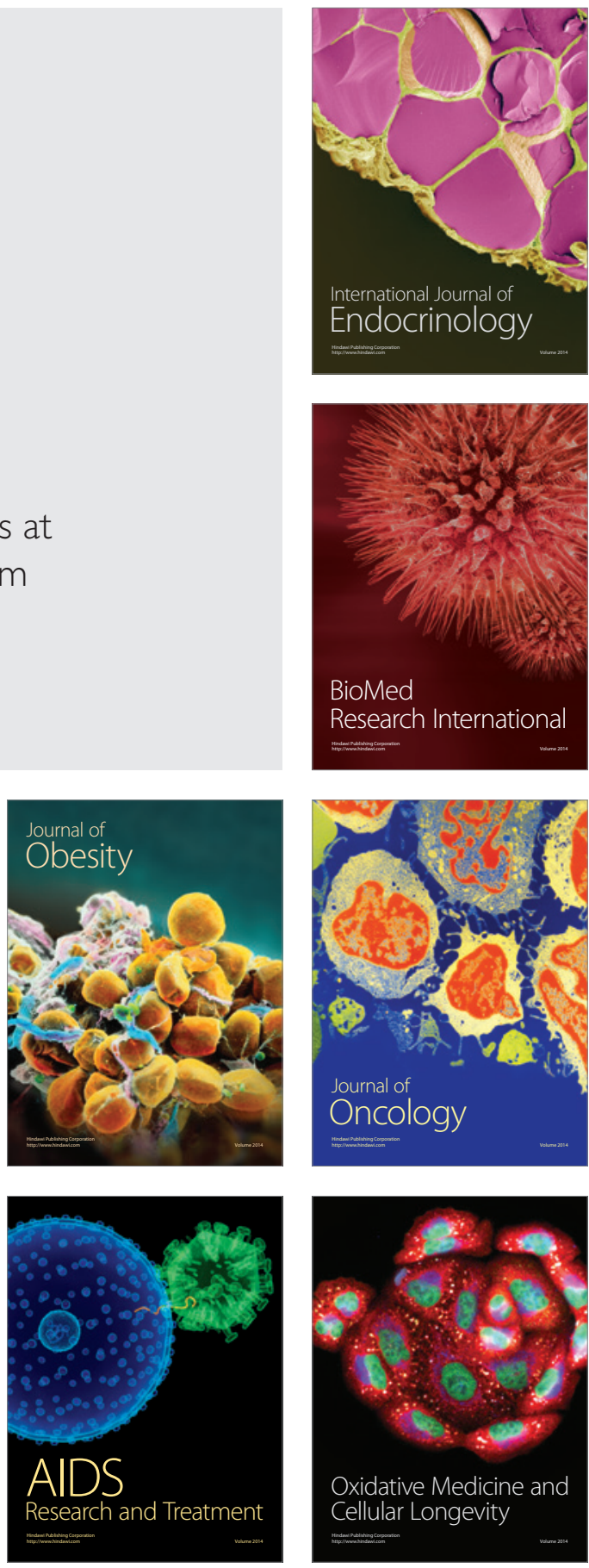